\title{
Synthesis of fused quinazolinethiones and their S-alkyl/aryl derivatives
}

\author{
Balbir Kaur* and Ramandeep Kaur \\ Department of Chemistry, Punjabi University, Patiala 147002, Punjab, India \\ E-mail: aries_balbir@yahoo.co.in
}

\begin{abstract}
Quinazoline derivatives are associated with broad spectrum of biological activities. In view of this, 4-substituted phenyl-3,4,5,6-tetrahydrobenzo[h]quinazoline-2(1H)-thiones were prepared under microwave irradiations through one-pot multicomponent reactions and these quinazolinethiones were then converted to S-alkyl/aryl quinazoline derivatives. The synthetic schemes of the prepared compounds are given.
\end{abstract}

Keywords: S-Alkylation of quinazolinethiones, condensation reaction, green chemistry, multicomponent reactions

\section{Introduction}

The quinazoline skeleton ${ }^{1}$ is of great importance to chemists as well as biologists as it is available in a large variety of naturally occurring compounds. It is also found in clinically useful molecules having diverse biological activities ${ }^{2}$ such as antiviral, antimalarial, anticonvulsant, antibacterial, diuretic, hypnotic, hypoglycaemic, antitumoral and antihypertensive. Literature study reveals that quinazoline-2(1H)-thiones have been prepared under thermal conditions through multistep reactions. However, as the concept of one-pot multicomponent reaction ${ }^{3}$ (MCR) and Microwave-induced Organic Reaction Enhancement (MORE) chemistry ${ }^{4,5,8}$ is gaining importance due to increasing environmental and economical concern, we have modified the synthetic route of quinazoline-2 $(1 H)$-thiones to one-pot multicomponent cyclocondensation reaction under microwave irradiations.

Through this modification, precious solvents can be saved, the reaction time can be reduced and overall yield has been improved by reducing the number of steps. Thus, it is a step towards green chemistry.

The present work also includes the reaction of the thiones with suitable reagents forming alkylated products. Literature survey reveals that 2-(substituted thio)-4-substituted phenyl- 
1,4,5,6-tetrahydrobenzo[ $h]$ quinazolines are unknown. Prompted by these observations, we report herein a general route to the title compounds.

\section{Results and Discussions}

The synthesis of 4-phenyl-3,4,5,6-tetrahydrobenzo[h]-quinazoline-2(1H)-thione $\mathbf{1}$ has been reported through double step reaction under thermal conditions ${ }^{6}$. However, to become more economical and environmental friendly, the synthetic route of compound $\mathbf{1}$ is being modified from double step to single step reaction under microwave irradiations. For this, the acidcatalysed condensation of tetralone, thiourea, and benzaldehyde was carried out taking acetonitrile as solvent at 30\% power level (Scheme 1). The results obtained demonstrate the versatility of the process as considerable rate enhancement has been observed.

Different aromatic aldehydes were examined under the optimized conditions (Table 1).
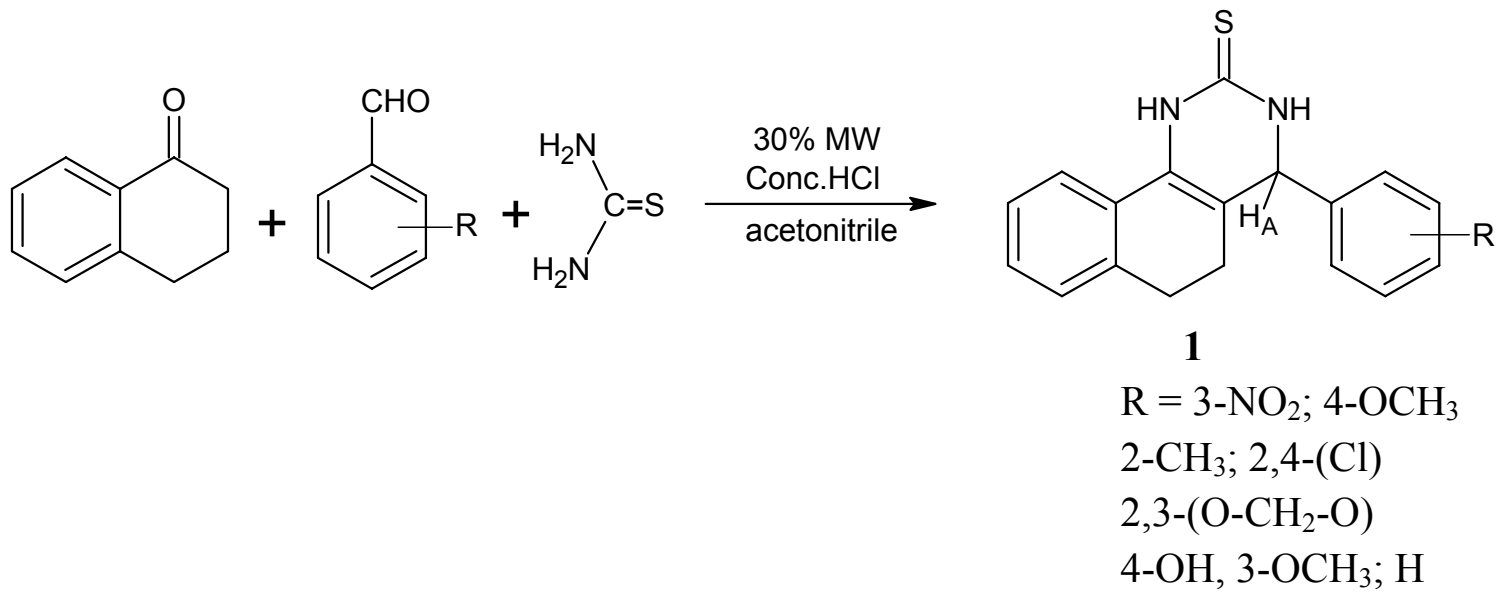

\section{Scheme 1}

Table 1. Synthesis of 4-substituted phenyl-3,4,5,6-tetrahydrobenzo[ $h]$-quinazoline-2(1H)thiones (1a-1g)

\begin{tabular}{cllcccc}
\hline Entry & \multicolumn{1}{c}{$\mathrm{R}$} & Time$(m i n)$. & Product & M.p $\left({ }^{0} \mathrm{C}\right)$ & Lit.m.p ${ }^{7}\left({ }^{0} \mathrm{C}\right)$ & Yield(\%) \\
\hline 1. & $3-\mathrm{NO}_{2}$ & 6.30 & $1 \mathrm{a}$ & $245-247$ & --- & 53 \\
2. & $4-\mathrm{OCH}_{3}$ & 5.00 & $1 \mathrm{~b}$ & $219-221$ & $220-22$ & 40 \\
3. & $2-\mathrm{CH}_{3}$ & 6.00 & $1 \mathrm{c}$ & $227-229$ & --- & 45 \\
4. & $2,4-(\mathrm{Cl})$ & 4.00 & $1 \mathrm{~d}$ & $217-219$ & ---- & 36 \\
5. & $2,3-\left(\mathrm{O}-\mathrm{CH}_{2}-\mathrm{O}\right)$ & 3.30 & $1 \mathrm{e}$ & $224-225$ & ---- & 47 \\
6. & $4-\mathrm{OH}, 3-\mathrm{OCH}_{3}$ & 3.30 & $1 \mathrm{f}$ & $211-213$ & ---- & 35 \\
7. & $\mathrm{H}$ & 5.30 & $1 \mathrm{~g}$ & $256-257$ & 256 & 46 \\
\hline
\end{tabular}


A variety of aromatic aldehydes carrying either electron-donating or electron-withdrawing sustituents reacted very well, giving products in high purity. Further these thiones can be alkylated and for this, a mixture of quinazolinethione, alkylating agent and ethanol was refluxed for 3-5 hrs. The reaction was monitored by TLC and work-up of the reaction yielded a compound 2 which was labeled as 2-(substituted thio)-4- substituted phenyl-1,4,5,6tetrahydrobenzo[h]quinazolines (Scheme 2).
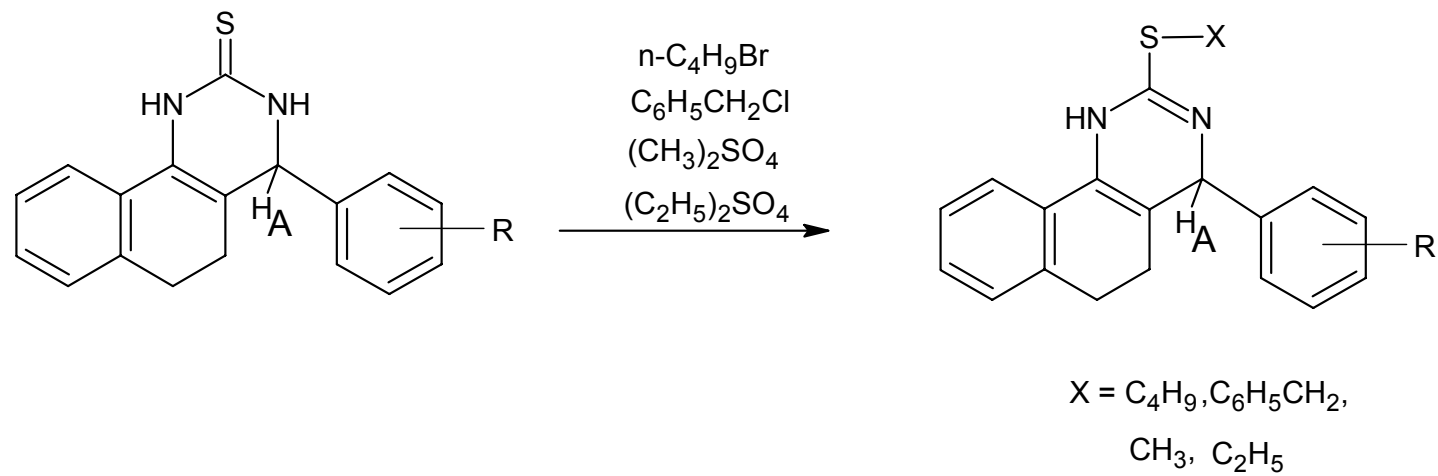

1

2

\section{Scheme 2}

Especially noteworthy is the survival of a variety of functional groups such as nitro, hydroxyl, methoxy, chloro, double bond etc. under the reaction conditions.

\section{Conclusions}

In summary, we have changed the methodology for the synthesis of quinazoline-2-thiones 1 to a three-component condensation reaction in one-pot, using microwave irradiations. By using the new methodology, the preparation of quinazoline-2-thione analogues is a simple, cost-effective, time-saving and eco-friendly process. Also, alkylated derivatives of compound $\mathbf{1}$ have been prepared.

\section{Experimental Section}

General Procedures. Melting points are uncorrected and were determined in open end capillaries. Thin layer chromatography was performed on Silica gel G (Merck). ${ }^{1} \mathrm{H}$ NMR spectra were recorded on BRUKER ADVANCE II 400 NMR Spectrometer. The IR spectra were recorded on Perkin-Elmer spectrum RX IFT-IR System. The mass Spectra were obtained on a JEOL 5x102/DA-6000 mass spectrometer. All the compounds gave satisfactory elemental 
analysis within $\pm 0.4 \%$ of theoretical values. The microwave irradiated reactions were performed in domestic household microwave oven Samsung M1777N.

Table 2. Synthesis of 2-(substituted thio)- 4-substituted phenyl-1,4,5,6tetrahydrobenzo[ $h]$ quinazolines $(\mathbf{2 a - 2 t})$

\begin{tabular}{cccccc}
\hline Entry & $\mathrm{R}$ & $\mathrm{X}$ & Product & M.p. ${ }^{0} \mathrm{C}$ & Yield \% \\
\hline 1. & $3-\mathrm{NO}_{2}$ & $\mathrm{C}_{2} \mathrm{H}_{5}$ & $2 \mathrm{a}$ & $79-81$ & 20 \\
2 & $4-\mathrm{OCH}_{3}$ & $\mathrm{C}_{2} \mathrm{H}_{5}$ & $2 \mathrm{~b}$ & $177-178$ & 40 \\
3 & $2,3-\left(\mathrm{O}-\mathrm{CH}_{2}-\mathrm{O}\right)$ & $\mathrm{C}_{2} \mathrm{H}_{5}$ & $2 \mathrm{c}$ & $173-175$ & 38 \\
4 & $4-\mathrm{OH}, 3-\mathrm{OCH}_{3}$ & $\mathrm{C}_{2} \mathrm{H}_{5}$ & $2 \mathrm{~d}$ & $98-100$ & 78 \\
5 & $\mathrm{H}$ & $\mathrm{C}_{2} \mathrm{H}_{5}$ & $2 \mathrm{e}$ & $208-210$ & 16 \\
6 & $4-\mathrm{OCH}$ & $\mathrm{CH}_{3}$ & $2 \mathrm{f}$ & $179-181$ & 38 \\
7 & $2,4-\left(\mathrm{Cl}_{3}\right.$ & $\mathrm{CH}_{3}$ & $2 \mathrm{~g}$ & $170-172$ & 31 \\
8 & $2,3-\left(\mathrm{O}-\mathrm{CH}_{2}-\mathrm{O}\right)$ & $\mathrm{CH}_{3}$ & $2 \mathrm{~h}$ & $186-187$ & 73 \\
9 & $4-\mathrm{OH}, 3-\mathrm{OCH}$ & $\mathrm{CH}_{3}$ & $2 \mathrm{i}$ & $150-152$ & 20 \\
10 & $\mathrm{H}$ & $\mathrm{CH}_{3}$ & $2 \mathrm{j}$ & $198-200$ & 45 \\
11 & $3-\mathrm{NO}_{2}$ & $\mathrm{C}_{6} \mathrm{H}_{5} \mathrm{CH}_{2}$ & $2 \mathrm{k}$ & $214-216$ & 65 \\
12 & $2,3-\left(\mathrm{O}-\mathrm{CH}_{2}-\mathrm{O}\right)$ & $\mathrm{C}_{6} \mathrm{H}_{5} \mathrm{CH}_{2}$ & $2 \mathrm{l}$ & $179-181$ & 53 \\
13 & $4-\mathrm{OCH}$ & $\mathrm{C}_{6} \mathrm{H}_{5} \mathrm{CH}_{2}$ & $2 \mathrm{~m}$ & $202-203$ & 60 \\
14 & $\mathrm{H}$ & $\mathrm{C}_{6} \mathrm{H}_{5} \mathrm{CH}_{2}$ & $2 \mathrm{n}$ & $218-219$ & 65 \\
15 & $2,4-\left(\mathrm{Cl}_{3}\right.$ & $\mathrm{C}_{4} \mathrm{H}_{9}$ & $2 \mathrm{o}$ & $177-179$ & 69 \\
16 & $4-\mathrm{OCH}$ & $\mathrm{C}_{4} \mathrm{H}_{9}$ & $2 \mathrm{p}$ & $89-91$ & 60 \\
17 & $2-\mathrm{CH}_{3}$ & $\mathrm{C}_{4} \mathrm{H}_{9}$ & $2 \mathrm{q}$ & $202-204$ & 85 \\
18 & $2,3-\left(\mathrm{O}-\mathrm{CH}_{2}-\mathrm{O}\right)$ & $\mathrm{C}_{4} \mathrm{H}_{9}$ & $2 \mathrm{r}$ & $199-201$ & 80 \\
19 & $4-\mathrm{OH}, 3-\mathrm{OCH}$ & $\mathrm{C}_{4} \mathrm{H}_{9}$ & $2 \mathrm{~s}$ & $83-85$ & 70 \\
20 & $4 \mathrm{H}$ & $\mathrm{C}_{4} \mathrm{H}_{9}$ & $2 \mathrm{t}$ & $219-221$ & 56 \\
\hline & & & &
\end{tabular}

General procedure for synthesis of 4-substituted phenyl-3,4,5,6-tetrahydrobenzo [h]quinazoline-2(1H)-thiones

A mixture of tetralone $(0.01 \mathrm{~mole}, 1.46 \mathrm{~g})$, thiourea $(0.01,0.76 \mathrm{~g})$ and substituted aromatic aldehyde $(0.01 \mathrm{~mole})$ were subjected to microwave heating for 3-7 minutes using acetonitrile $(5 \mathrm{ml})$ as energy transfer medium and $\mathrm{HCl}(0.5 \mathrm{ml})$ as a catalyst. The reaction mixture on standing for few hours afforded product which was filtered under reduced pressure and recrystallised out of alcohol. The products were characterized on the basis of m.pts., IR, NMR, mass and elemental analysis spectra. The spectral data is given below .

4-(3-Nitrophenyl)-3,4,5,6-tetrahydrobenzo $[h]$ quinazoline-2(1H)-thione (1a). $\operatorname{IR}\left(\mathrm{KBr}, \mathrm{cm}^{-1}\right)$

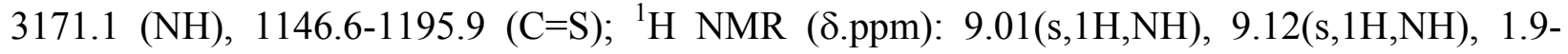
2.2(t,2H, $\mathrm{C}_{5} \mathrm{CH}_{2}$ or $\left.\mathrm{C}_{6} \mathrm{CH}_{2}\right), \quad 2.75-2.81\left(\mathrm{t}, 2 \mathrm{H}, \mathrm{C}_{5} \mathrm{CH}_{2}\right.$ or $\left.\mathrm{C}_{6} \mathrm{CH}_{2}\right), \quad 5.19\left(\mathrm{~s}, 1 \mathrm{H}, \mathrm{H}_{\mathrm{A}}\right), \quad 7.30-8.27$ (m,8H,Ar-H); Anal. Calcd for $\mathrm{C}_{18} \mathrm{H}_{15} \mathrm{~N}_{3} \mathrm{SO}_{2}$ : C 64.09; H, 4.45; N, 12.46. Found: C, 64.01; H, 4.39; N, $12.40 \%$ 
4-(4-Methoxyphenyl)-3,4,5,6-tetrahydrobenzo[h]quinazoline-2(1H)-thione (1b). IR ( $\mathrm{KBr}, \mathrm{cm}^{-}$

$\left.{ }^{1}\right): 3195(\mathrm{NH}), \quad 1171(\mathrm{C}=\mathrm{S}) ;{ }^{1} \mathrm{H} \mathrm{NMR}(\delta, \mathrm{ppm}): 7.82(\mathrm{~s}, 1 \mathrm{H}, \mathrm{NH}), 1.9-2,09\left(\mathrm{t}, 2 \mathrm{H}, \mathrm{C}_{5} \mathrm{CH}_{2}\right.$ or $\left.\mathrm{C}_{6} \mathrm{CH}_{2}\right), 2.67-2.77\left(\mathrm{t}, 2 \mathrm{H}, \mathrm{C}_{5} \mathrm{CH}_{2}\right.$ or $\left.\mathrm{C}_{6} \mathrm{CH}_{2}\right), 5.04\left(\mathrm{~s}, 1 \mathrm{H}, \mathrm{H}_{\mathrm{A}}\right), 3.77\left(\mathrm{~s}, 3 \mathrm{H}, \mathrm{OCH}_{3}\right), 6.85-7.28(\mathrm{~m}, 9 \mathrm{H}$, Ar-H \& NH ); Mass (m/z) : 322 (63.32\%) M+, 262(15.97\%), 215(100\%), 181 (19.71\%), 108 (13.44\%); Anal. Calcd for $\mathrm{C}_{19} \mathrm{H}_{18} \mathrm{~N}_{2} \mathrm{SO}$ : C 70.80; H, 5.59; N, 8.69. Found: C, 70.62; H, 5.32; N, $8.49 \%$

4-(2-Methylphenyl)-3,4,5,6-tetrahydrobenzo[ $h]$ quinazoline-2(1H)-thione (1c). IR $\left(\mathrm{KBr}, \mathrm{cm}^{-1}\right)$ : $3210.1(\mathrm{NH}), 1193.8(\mathrm{C}=\mathrm{S}) ;{ }^{1} \mathrm{H}$ NMR $(\delta, \mathrm{ppm}): 6.57$ (s,1H,NH), $7.73(\mathrm{~s}, 1 \mathrm{H}, \mathrm{NH}), 1.89-2.06$ $\left(\mathrm{t}, 2 \mathrm{H}, \mathrm{C}_{5} \mathrm{CH}_{2}\right.$ or $\left.\mathrm{C}_{6} \mathrm{CH}_{2}\right), 2.70-2.79\left(\mathrm{t}, 2 \mathrm{H}, \mathrm{C}_{5} \mathrm{CH}_{2}\right.$ or $\left.\mathrm{C}_{6} \mathrm{CH}_{2}\right), 5.44\left(\mathrm{~s}, 1 \mathrm{H}, \mathrm{H}_{\mathrm{A}}\right), 2.43\left(\mathrm{~s}, 3 \mathrm{H}, \mathrm{CH}_{3}\right), 7.16-$ 7.33(m,8H,Ar-H) Anal. Calcd for $\mathrm{C}_{19} \mathrm{H}_{18} \mathrm{~N}_{2} \mathrm{~S}: \mathrm{C}$ 74.51; H, 5.88; N, 9.15. Found: C, 74.23; H, $5.62 ; \mathrm{N}, 8.99 \%$

4-(2,4-Dichlorophenyl)-3,4,5,6-tetrahydrobenzo[h]quinazoline-2(1H)-thione $\quad(1 \mathrm{~d}) . \quad$ IR $\left(\mathrm{KBr}, \mathrm{cm}^{-1}\right)$ : $3190.8(\mathrm{NH}), 1140.2-1198.4(\mathrm{C}=\mathrm{S}) ;{ }^{1} \mathrm{H}$ NMR $(\delta, \mathrm{ppm}): 6.88(\mathrm{~s}, 1 \mathrm{H}, \mathrm{NH}), 7.84$ $(\mathrm{s}, 1 \mathrm{H}, \mathrm{NH}), 1.97-2.22\left(\mathrm{t}, 2 \mathrm{H}, \mathrm{C}_{5} \mathrm{CH}_{2}\right.$ or $\left.\mathrm{C}_{6} \mathrm{CH}_{2}\right), 2.75-2.84\left(\mathrm{t}, 2 \mathrm{H}, \mathrm{C}_{5} \mathrm{CH}_{2}\right.$ or $\left.\mathrm{C}_{6} \mathrm{CH}_{2}\right)$, 5.62-5.63 $\left(\mathrm{s}, 1 \mathrm{H}, \mathrm{H}_{\mathrm{A}}\right), 7.19-7.43(\mathrm{~m}, 7 \mathrm{H}, \mathrm{Ar}-\mathrm{H})$;Anal. Calcd for $\mathrm{C}_{18} \mathrm{H}_{14} \mathrm{~N}_{2} \mathrm{SCl}_{2}$ : C 59.83; H, 3.88; N, 7.75. Found: C, 59.53; H, 3.56; N, 7.59\%

4-(2,3-Methylenedioxyphenyl)-3,4,5,6-tetrahydrobenzo $[h] q u i n a z o l i n e-2(1 H)$-thione (1e). IR $\left(\mathrm{KBr}, \mathrm{cm}^{-1}\right)$ : 3201.6(NH), $1137.9-1195.9(\mathrm{C}=\mathrm{S}) ;{ }^{1} \mathrm{H}$ NMR $(\delta, \mathrm{ppm}): 8.18$ (s,1H,NH), 8.36 $(\mathrm{s}, 1 \mathrm{H}, \mathrm{NH}), \quad 1.97-2.11\left(\mathrm{t}, 2 \mathrm{H}, \mathrm{C}_{5} \mathrm{CH}_{2}\right.$ or $\left.\mathrm{C}_{6} \mathrm{CH}_{2}\right), 2.69-2.78\left(\mathrm{t}, 2 \mathrm{H}, \mathrm{C}_{5} \mathrm{CH}_{2}\right.$ or $\left.\mathrm{C}_{6} \mathrm{CH}_{2}\right), 4.95$ $\left(\mathrm{s}, 1 \mathrm{H}, \mathrm{H}_{\mathrm{A}}\right)$, 5.94-5.95 (s, 2H, O-CH2-O ), 6.74-7.45 (m,7H,Ar-H); Mass (m/z): $336(90.92 \%) \mathrm{M}^{+}$ ,215 (99.04\%), 260 (2.29\%), 76(56.81\%), 260 (2.29\%),

Anal. Calcd for $\mathrm{C}_{19} \mathrm{H}_{16} \mathrm{~N}_{2} \mathrm{SO}_{2}$ : C 67.86; H, 4.76; N, 8.33. Found: C, 67.55; H, 4.82; N, 8.12\% 4-(4-Hydroxy-3-methoxyphenyl)-3,4,5,6-tetrahydrobenzo[h]quinazoline-2(1H)-thione (1f). IR $\left(\mathrm{KBr}, \mathrm{cm}^{-1}\right)$ : 3104.1-3176.9 (NH), 1122.2-1195.4 (C=S), $3346.9(\mathrm{O}-\mathrm{H}){ }^{1} \mathrm{H}$ NMR $(\delta, p p m)$ : 10.76 ( s,1H,NH ), 10.9 (s,1H,NH), 6.62-7.57 (m,7H,Ar-H),1.91-2.19 (t,2H,C $\mathrm{CH}_{2}$ or $\left.\mathrm{C}_{6} \mathrm{CH}_{2}\right)$, 2.49-2.58 (t,2H, $\mathrm{C}_{5} \mathrm{CH}_{2}$ or $\left.\mathrm{C}_{6} \mathrm{CH}_{2}\right), 5.03\left(\mathrm{~s} 1 \mathrm{H}, \mathrm{H}_{\mathrm{A}}\right), .10(\mathrm{~s} 1 \mathrm{H}, \mathrm{OH}), 3.71\left(\mathrm{~s}, 3 \mathrm{H}, \mathrm{OCH}_{3}\right)$; Anal. Calcd for $\mathrm{C}_{19} \mathrm{H}_{18} \mathrm{~N}_{2} \mathrm{SO}_{2}$ : C 67.45; H, 5.32; N, 8.28. Found: C, 67.56; H, 5.76; N, 8.01\%

4-Phenyl-3,4,5,6-tetrahydrobenzo[h]quinazoline-2(1H)-thione (1g). IR $\left(\mathrm{KBr}, \mathrm{cm}^{-1}\right): 3157.3$ $(\mathrm{NH}), 1195.3(\mathrm{C}=\mathrm{S}) ;{ }^{1} \mathrm{H}$ NMR $(\delta, \mathrm{ppm}): 8.56(\mathrm{~s}, 1 \mathrm{H}, \mathrm{NH}), 8.68(\mathrm{~s}, 1 \mathrm{H}, \mathrm{NH}), 1.89-2.1\left(\mathrm{t}, 2 \mathrm{H}, \mathrm{C}_{5} \mathrm{CH}_{2}\right.$ or $\left.\mathrm{C}_{6} \mathrm{CH}_{2}\right), 2.61-2.62\left(\mathrm{t}, 2 \mathrm{H}, \mathrm{C}_{5} \mathrm{CH}_{2}\right.$ or $\left.\mathrm{C}_{6} \mathrm{CH}_{2}\right), 5.01\left(\mathrm{~s}, 1 \mathrm{H}, \mathrm{H}_{\mathrm{A}}\right), 7.12-7.85(\mathrm{~m}, 7 \mathrm{H}, \mathrm{Ar}-\mathrm{H})$. Anal. Calcd for $\mathrm{C}_{18} \mathrm{H}_{16} \mathrm{~N}_{2} \mathrm{~S}$ : C 73.97; H, 5.48; N, 9.58. Found: C, 73.72; H, 5.56; N, 9.34\%

General procedure for synthesis of 2 -(ethyl/methyl thio)-4-substituted phenyl-1,4,5,6-tetrahydrobenzo[h]quinazolines

To quinazolinethione 1 (0.004 mole) dissolved in ethanol was added $\mathrm{NaOH}$ solution which was prepared by dissolving $\mathrm{NaOH}(0.160 \mathrm{~g})$ in water $(2 \mathrm{ml})$. The mixture was cooled. To this mixture diethyl sulphate $(0.004$ mole $)$ or dimethyl sulphate $(0.004$ mole $)$ was added dropwise while stirring the reaction mixture continuously. Then the reaction mixture was refluxed for $3 \mathrm{hrs}$. The reacton mixture was cooled and poured over crushed ice. Solid separated was filtered under 
reduced pressure, dried and recrystallised from ethanol to give $2 \mathrm{a}-2 \mathrm{j}$. The spectral data are given in table 3.

\section{General procedure for synthesis of 2 -(benzyl thio)-4-substituted phenyl-1,4,5,6-tetrahydro- benzo[h]quinazolines}

To quinazolinethione 1 (0.004 mole) dissolved in alcohol $(5 \mathrm{ml})$ was added benzyl chloride $(0,004$ mole $)$ and the reaction mixture was refluxed for $5 \mathrm{hrs}$. The mixture was cooled at room temperature. The solid separated was filtered and recrystallised from ethanol to give $2 \mathrm{k}-2 \mathrm{n}$. The spectral data is given in table 3 .

\section{General procedure for synthesis of 2-(butyl thio)-4-substituted phenyl-1,4,5,6-tetrahydro- benzo[h]quinazolines}

A mixture of powdered quinazolinethione 1 (0.004 mole), butyl bromide (0.004 mole) and absolute alcohol $(5 \mathrm{ml})$ was refluxed for $5 \mathrm{hrs}$. Then the product was allowed to separate at room temperature. After a long standing of 36-40 hrs., the product separated was filtered under reduced pressure and recrystallised from ethanol to give 2o-2t. The spectral data is given in table 3.

Table 3. Characterization data of the synthesized compounds

\begin{tabular}{|c|c|}
\hline Comp. & ${ }^{1} \mathrm{H}$ NMR $(\delta$, ppm); Mass, Elemental analysis \\
\hline $2 \mathrm{a}$ & $\begin{array}{l}\text { 9.58(s,1H,NH), 7.07-8.21(m,8H,Ar-H), } 5.03\left(\mathrm{~s}, 1 \mathrm{H}, \mathrm{H}_{\mathrm{A}}\right), 2.58-2.69\left(\mathrm{t}, 2 \mathrm{H}, \mathrm{C}_{5} \mathrm{CH}_{2} \text { or }\right. \\
\left.\mathrm{C}_{6} \mathrm{CH}_{2}\right), 1.89-2.21\left(\mathrm{t}, 2 \mathrm{H}, \mathrm{C}_{5} \mathrm{CH}_{2} \text { or } \mathrm{C}_{6} \mathrm{CH}_{2}\right), 2.92-3.04\left(\mathrm{t}, 3 \mathrm{H}, \mathrm{S}-\mathrm{CH}_{2}-\mathrm{CH}_{3}\right) .3 .20-3.29 \\
\left(\mathrm{q}, 1 \mathrm{H} \text { of } \mathrm{S}-\mathrm{CH}_{2}\right), 3.09-3.15\left(\mathrm{q}, 1 \mathrm{H} \text { of } \mathrm{S}-\mathrm{CH}_{2}\right) \text {. Anal. Calcd for } \mathrm{C}_{20} \mathrm{H}_{19} \mathrm{~N}_{3} \mathrm{SO}_{2}: \mathrm{C} \\
65.75 ; \mathrm{H}, 5.20 ; \mathrm{N}, 11.50 \text {. Found: } \mathrm{C}, 65.42 ; \mathrm{H}, 5.67 ; \mathrm{N}, 11.23 \% \text {. }\end{array}$ \\
\hline $2 b$ & $\begin{array}{l}7.82(\mathrm{~s}, 1 \mathrm{H}, \mathrm{NH}), 6.85-7.28(\mathrm{~m}, 8 \mathrm{H}, \mathrm{Ar}-\mathrm{H}), 5.04\left(\mathrm{~s}, 1 \mathrm{H}, \mathrm{H}_{\mathrm{A}}\right), 3.77\left(\mathrm{~s}, 3 \mathrm{H}, \mathrm{OCH}_{3}\right), 2.65- \\
2.77\left(\mathrm{t}, 2 \mathrm{H}, \mathrm{C}_{5} \mathrm{CH}_{2} \text { or } \mathrm{C}_{6} \mathrm{CH}_{2}\right), 1.90-2.09\left(\mathrm{t}, 2 \mathrm{H}, \mathrm{C}_{5} \mathrm{CH}_{2} \text { or } \mathrm{C}_{6} \mathrm{CH}_{2}\right), 3.24-3.30(\mathrm{q}, 1 \mathrm{H} \\
\left.\text { of } \mathrm{S}-\mathrm{CH}_{2}\right), 3.11-3.16\left(\mathrm{q}, 1 \mathrm{H} \text { of } \mathrm{S}-\mathrm{CH}_{2}\right), 2.9-3.03\left(\mathrm{t}, 3 \mathrm{H}, \mathrm{S}-\mathrm{CH}_{2}-\mathrm{CH}_{3}\right) \text {. Anal. Calcd for } \\
\mathrm{C}_{21} \mathrm{H}_{22} \mathrm{~N}_{2} \mathrm{SO}: \mathrm{C} 72 ; \mathrm{H}, 6.28 ; \mathrm{N}, 8 \text {. Found: } \mathrm{C}, 72.23 ; \mathrm{H}, 6.32 ; \mathrm{N}, 8.10 \% \text {. }\end{array}$ \\
\hline $2 \mathrm{c}$ & $\begin{array}{l}8.67(\mathrm{~s}, 1 \mathrm{H}, \mathrm{NH}), 6.74-7.58(\mathrm{~m}, 7 \mathrm{H}, \mathrm{Ar}-\mathrm{H}), 5.94-5.95\left(\mathrm{~s}, 2 \mathrm{H}, \mathrm{O}-\mathrm{CH}_{2}-\mathrm{O}\right), 4.92-4.93 \\
\left(\mathrm{~s}, 1 \mathrm{H}, \mathrm{H}_{\mathrm{A}}\right), 2.68-2.77\left(\mathrm{t}, 2 \mathrm{H}, \mathrm{C}_{5} \mathrm{CH}_{2} \text { or } \mathrm{C}_{6} \mathrm{CH}_{2}\right), 1.96-2.12\left(\mathrm{t}, 2 \mathrm{H}, \mathrm{C}_{5} \mathrm{CH}_{2} \text { or } \mathrm{C}_{6} \mathrm{CH}_{2}\right) \\
\text { 3.21-3.29 }\left(\mathrm{q}, 1 \mathrm{H} \text { of } \mathrm{S}_{-} \mathrm{CH}_{2}\right), 3.58-3.62\left(\mathrm{q}, 1 \mathrm{H} \text { of } \mathrm{S}-\mathrm{CH}_{2}\right), 3.04\left(\mathrm{t}, 3 \mathrm{H}, \mathrm{S}-\mathrm{CH}_{2}-\mathrm{CH}_{3}\right) \text {. } \\
\text { Anal. Calcd for } \mathrm{C}_{21} \mathrm{H}_{21} \mathrm{~N}_{2} \mathrm{SO}_{2}: \mathrm{C} 69.23 ; \mathrm{H}, 5.77 ; \mathrm{N}, 7.69 \text {. Found: C, 69.29; } \mathrm{H}, 5.74 ; \\
\text { N,7.71\%. }\end{array}$ \\
\hline $2 d$ & $\begin{array}{l}10.8(\mathrm{~s}, 1 \mathrm{H}, \mathrm{NH}), 8.10(\mathrm{~s}, 1 \mathrm{H}, \mathrm{OH}), 6.61-7.52(\mathrm{~m}, 7 \mathrm{H}, \mathrm{Ar}-\mathrm{H}), 5.03\left(\mathrm{~s}, 1 \mathrm{H}, \mathrm{H}_{\mathrm{A}}\right), 2.48- \\
2.57\left(\mathrm{t}, 2 \mathrm{H}, \mathrm{C}_{5} \mathrm{CH}_{2} \text { or } \mathrm{C}_{6} \mathrm{CH}_{2}\right), 1.92-2.21\left(\mathrm{t}, 2 \mathrm{H}, \mathrm{C}_{5} \mathrm{CH}_{2} \text { or } \mathrm{C}_{6} \mathrm{CH}_{2}\right), 3.23-3.29,(\mathrm{q}, 1 \mathrm{H} \text { of } \\
\left.\mathrm{S}-\mathrm{CH}_{2}\right), 3.16-3.19 \quad\left(\mathrm{q}, 1 \mathrm{H} \text { of } \mathrm{S}-\mathrm{CH}_{2}\right), 2.98-3.01 \quad\left(\mathrm{t}, 3 \mathrm{H}, \mathrm{S}-\mathrm{CH}_{2}-\mathrm{CH}_{3}\right), 3.70 \\
\left(\mathrm{~s}, 3 \mathrm{H}, \mathrm{OCH}_{3}\right) . \text { Anal. Calcd for } \mathrm{C}_{21} \mathrm{H}_{23} \mathrm{~N}_{2} \mathrm{SO}_{2}: \mathrm{C} 68.85 ; \mathrm{H}, 6.28 ; \mathrm{N}, 7.65 . \text { Found: C, } \\
68.52 ; \mathrm{H}, 6.32 ; \mathrm{N}, 7.89 \% \text {. }\end{array}$ \\
\hline
\end{tabular}


2e $\quad 11.99(\mathrm{~s}, 1 \mathrm{H}, \mathrm{NH}), 7.15-7.36(\mathrm{~m}, 9 \mathrm{H}, \mathrm{Ar}-\mathrm{H}), 5.42\left(\mathrm{~s}, 1 \mathrm{H}, \mathrm{H}_{\mathrm{A}}\right), 2.76-2.78,\left(\mathrm{t}, 2 \mathrm{H}, \mathrm{C}_{5} \mathrm{CH}_{2}\right.$ or $\mathrm{C}_{6} \mathrm{CH}_{2}$ ), 1.89-2.17 (t,2H, $\mathrm{C}_{5} \mathrm{CH}_{2}$ or $\mathrm{C}_{6} \mathrm{CH}_{2}$ ), 3.21-3.30 (q,1H of S- $\left.\mathrm{CH}_{2}\right), 3.10-3.17$ (q,1H of S-CH $\mathrm{CH}_{2}$, 2.81-3.01 (t,3H,S-CH$\left.-\mathrm{CH}_{3}\right)$. Anal. Calcd for $\mathrm{C}_{20} \mathrm{H}_{20} \mathrm{~N}_{2} \mathrm{~S}: \mathrm{C}$ 75; $\mathrm{H}, 6.25 ; \mathrm{N}, 8.75$. Found: C, 74.98; H, 6.02; N, 8.43\%.

$7.82(\mathrm{~s}, 1 \mathrm{H}, \mathrm{NH}), 6.86-7.30(\mathrm{~m}, 8 \mathrm{H}, \mathrm{Ar}-\mathrm{H}), 5.01\left(\mathrm{~s}, 1 \mathrm{H}, \mathrm{H}_{\mathrm{A}}\right), 3.77\left(\mathrm{~s}, 3 \mathrm{H}, \mathrm{OCH}_{3}\right), 2.63-$ $2.81\left(\mathrm{t}, 2 \mathrm{H}, \mathrm{C}_{5} \mathrm{CH}_{2}\right.$ or $\left.\mathrm{C}_{6} \mathrm{CH}_{2}\right), 1.84-2.11\left(\mathrm{t}, 2 \mathrm{H}, \mathrm{C}_{5} \mathrm{CH}_{2}\right.$ or $\left.\mathrm{C}_{6} \mathrm{CH}_{2}\right), 2.98\left(\mathrm{~s}, 3 \mathrm{H}, \mathrm{S}-\mathrm{CH}_{3}\right)$ Anal. Cald for $\mathrm{C}_{20} \mathrm{H}_{20} \mathrm{~N}_{2} \mathrm{SO}: \mathrm{C} 71.43 ; \mathrm{H}, 5.95 ; \mathrm{N}, 8.33$. Found: C, 71.67; H, $5.53 ; \mathrm{N}, 8.10 \%$.

$2 \mathrm{~g} \quad 7.84(\mathrm{~s}, 1 \mathrm{H}, \mathrm{NH}), 7.19-7.50(\mathrm{~m}, 7 \mathrm{H}, \mathrm{Ar}-\mathrm{H}), 5.62\left(\mathrm{~s}, 1 \mathrm{H}, \mathrm{H}_{\mathrm{A}}\right), 2.75-2.84\left(\mathrm{t}, 2 \mathrm{H}, \mathrm{C}_{5} \mathrm{CH}_{2}\right.$ or $\left.\mathrm{C}_{6} \mathrm{CH}_{2}\right), 2.66\left(\mathrm{~s}, 3 \mathrm{H}, \mathrm{S}-\mathrm{CH}_{3}\right), 1.97-2.20\left(\mathrm{t}, 2 \mathrm{H}, \mathrm{C}_{5} \mathrm{CH}_{2}\right.$ or $\left.\mathrm{C}_{6} \mathrm{CH}_{2}\right)$. Anal. Calcd for $\mathrm{C}_{19} \mathrm{H}_{17} \mathrm{~N}_{2} \mathrm{SCl}$ : C, 60.80; H, 4.53; N, 7.47. Found: C, 60.65; H, 4.62; N, 7.39\%.

$2 \mathrm{~h}$ $8.82(\mathrm{~s}, 1 \mathrm{H}, \mathrm{NH}), 6.74-7.65(\mathrm{~m}, 7 \mathrm{H}, \mathrm{Ar}-\mathrm{H}), 4.91\left(\mathrm{~s}, 1 \mathrm{H}, \mathrm{H}_{\mathrm{A}}\right), 5.94-5.95\left(\mathrm{~s}, 2 \mathrm{H}, \mathrm{O}^{-} \mathrm{CH}_{2-}\right.$ O), 2.67-2.77 (t, $2 \mathrm{H}, \mathrm{C}_{5} \mathrm{CH}_{2}$ or $\mathrm{C}_{6} \mathrm{CH}_{2}$ ), 1.96-2.12 (t, $2 \mathrm{H}, \mathrm{C}_{5} \mathrm{CH}_{2}$ or $\mathrm{C}_{6} \mathrm{CH}_{2}$ ), 3.13 (s,3H, S-CH $)_{3}$ m/z $350(0.27 \%) \mathrm{M}^{+}, 335(76.34 \%), 215$ (100\%), 88 (20.67\%). Anal. Calcd for $\mathrm{C}_{20} \mathrm{H}_{19} \mathrm{~N}_{2} \mathrm{SO}_{2}: \mathrm{C}, 68.57 ; \mathrm{H}, 5.43 ; \mathrm{N}, 8$. Found: C, 68.54; $\mathrm{H}$, $5.36 ; \mathrm{N}, 8.13 \%$.

$2 \mathrm{i} \quad 10.8(\mathrm{~s}, 1 \mathrm{H}, \mathrm{NH}), 8.21(\mathrm{~s}, 1 \mathrm{H}, \mathrm{OH}), 6.62-7.54(\mathrm{~m}, 7 \mathrm{H}, \mathrm{Ar}-\mathrm{H}), 5.02\left(\mathrm{~s}, 1 \mathrm{H}, \mathrm{H}_{\mathrm{A}}\right), 2.45-$ $2.54\left(\mathrm{t}, 2 \mathrm{H}, \mathrm{C}_{5} \mathrm{CH}_{2}\right.$ or $\left.\mathrm{C}_{6} \mathrm{CH}_{2}\right), 1.90-2.19\left(\mathrm{t}, 2 \mathrm{H}, \mathrm{C}_{5} \mathrm{CH}_{2}\right.$ or $\left.\mathrm{C}_{6} \mathrm{CH}_{2}\right), 3.11(\mathrm{~s}, 3 \mathrm{H}, \mathrm{S}-$ $\left.\mathrm{CH}_{3}\right)$, 3.72(s,3H, $\left.\mathrm{OCH}_{3}\right)$. Anal. Calcd for $\mathrm{C}_{20} \mathrm{H}_{21} \mathrm{~N}_{2} \mathrm{SO}_{2}$ : C 68.18; H, 5.96; N, 7.95. Found: C, 68.38; H, 5.47; N, 8.01\%.

$2 \mathrm{j} \quad 8.42(\mathrm{~s}, 1 \mathrm{H}, \mathrm{NH}), 7.13-7.47(\mathrm{~m}, 9 \mathrm{H}, \mathrm{Ar}-\mathrm{H}), 5.03\left(\mathrm{~s}, 1 \mathrm{H}, \mathrm{H}_{\mathrm{A}}\right), 2.67-2.84\left(\mathrm{t}, 2 \mathrm{H}, \mathrm{C}_{5} \mathrm{CH}_{2}\right.$ or $\left.\mathrm{C}_{6} \mathrm{CH}_{2}\right), 1.90-2.16\left(\mathrm{t}, 2 \mathrm{H}, \mathrm{C}_{5} \mathrm{CH}_{2}\right.$ or $\left.\mathrm{C}_{6} \mathrm{CH}_{2}\right), 2.75\left(\mathrm{~s}, 3 \mathrm{H}, \mathrm{S}-\mathrm{CH}_{3}\right)$. Anal. Calcd for $\mathrm{C}_{19} \mathrm{H}_{18} \mathrm{~N}_{2} \mathrm{~S}: \mathrm{C} 74.51 ; \mathrm{H}, 5.88 ; \mathrm{N}, 9.15$. Found: C, 74.78; H, 5.32; N, 9.34\%.

$2 \mathrm{k} \quad 11.95(\mathrm{~s}, 1 \mathrm{H}, \mathrm{NH}), 7.02-8.21(\mathrm{~m}, 13 \mathrm{H}, \mathrm{Ar}-\mathrm{H}), 5.61\left(\mathrm{~s}, 1 \mathrm{H}, \mathrm{H}_{\mathrm{A}}\right), 5.00-5.04(\mathrm{~d}, 1 \mathrm{H}$ of S$\left.\mathrm{CH}_{2}\right)$, 4.39-4.43 (d,1H of S-CH$), 2.65-2.9\left(\mathrm{t}, 2 \mathrm{H}, \mathrm{C}_{5} \mathrm{CH}_{2}\right.$ or $\left.\mathrm{C}_{6} \mathrm{CH}_{2}\right), 1.9-2.39$ $\left(\mathrm{t}, 2 \mathrm{H}, \mathrm{C}_{5} \mathrm{CH}_{2}\right.$ or $\left.\mathrm{C}_{6} \mathrm{CH}_{2}\right) \mathrm{m} / \mathrm{z} 427(4.09 \%) \mathrm{M}^{+}, 337(1.35 \%), 305$ (11.53\%), 91(100\%). Anal. Calcd for $\mathrm{C}_{25} \mathrm{H}_{21} \mathrm{~N}_{3} \mathrm{SO}_{2}$ : C, 70.26; H, 4.92; N, 9.85. Found: $\mathrm{C}$, $70.29 ; \mathrm{H}, 4.94 ; \mathrm{N}, 9.88 \%$.

$21 \quad 11.32(\mathrm{~s}, 1 \mathrm{H}, \mathrm{NH}), 6.73-7.61(\mathrm{~m}, 12 \mathrm{H}, \mathrm{Ar}-\mathrm{H}), 5.95-5.96\left(\mathrm{~s}, 2 \mathrm{H}, \mathrm{O}-\mathrm{CH}_{2}-\mathrm{O}\right), 5.25$ $\left(\mathrm{s}, 1 \mathrm{H}, \mathrm{H}_{\mathrm{A}}\right), 4.65-4.71(\mathrm{~d}, 1 \mathrm{H}$ of S-CH$), 4.38-4.42\left(\mathrm{~d}, 1 \mathrm{H}\right.$ of S-CH $\left.\mathrm{CH}_{2}\right), 2.65-2.76(\mathrm{t}, 2 \mathrm{H}$, $\mathrm{C}_{5} \mathrm{CH}_{2}$ or $\left.\mathrm{C}_{6} \mathrm{CH}_{2}\right)$, 1.99-2.14 (t, $2 \mathrm{H}_{2} \mathrm{C}_{5} \mathrm{CH}_{2}$ or $\mathrm{C}_{6} \mathrm{CH}_{2}$ ). Anal. Calcd for $\mathrm{C}_{26} \mathrm{H}_{23} \mathrm{~N}_{2} \mathrm{SO}_{2}$ : C 73.24; H, 5.39; N, 6.57. Found: C, 73.36; H, 5.48; N, 6.34\%.

$2 \mathrm{~m} \quad 11.61(\mathrm{~s}, 1 \mathrm{H}, \mathrm{NH}), 6.82-7.71(\mathrm{~m}, 13 \mathrm{H}, \mathrm{Ar}-\mathrm{H}), 5.36\left(\mathrm{~s}, 1 \mathrm{H}, \mathrm{H}_{\mathrm{A}}\right), 4.92-4.96(\mathrm{~d}, 1 \mathrm{H}$ of S$\left.\mathrm{CH}_{2}\right), 4.42-4.46(\mathrm{~d}, 1 \mathrm{H}$ of S-CH$), 3.81\left(\mathrm{~s}, 3 \mathrm{H}, \mathrm{OCH}_{3}\right), 2.73-2.78\left(\mathrm{t}, 2 \mathrm{H}, \mathrm{C}_{5} \mathrm{CH}_{2}\right.$ or $\mathrm{C}_{6} \mathrm{CH}_{2}$ ), 2.02-2.23 (t, $2 \mathrm{H}, \mathrm{C}_{5} \mathrm{CH}_{2}$ or $\mathrm{C}_{6} \mathrm{CH}_{2}$ ) Anal. Calcd for $\mathrm{C}_{26} \mathrm{H}_{24} \mathrm{~N}_{2} \mathrm{SO}: \mathrm{C}$, 75.73; $\mathrm{H}, 5.82 ; \mathrm{N}, 6.79$. Found: $\mathrm{C}, 75.76 ; \mathrm{H}, 5.88 ; \mathrm{N}, 6.76 \%$.

2n $\quad 11.79(\mathrm{~s}, 1 \mathrm{H}, \mathrm{NH}), 7.13-7.68(\mathrm{~m}, 14 \mathrm{H}, \mathrm{Ar}-\mathrm{H}), 5.40\left(\mathrm{~s}, 1 \mathrm{H}, \mathrm{H}_{\mathrm{A}}\right), 4.87-4.92(\mathrm{~d}, 1 \mathrm{H}$ of S$\left.\mathrm{CH}_{2}\right), 4.46-4.50(\mathrm{~d}, 1 \mathrm{H}$ of S-CH$), 2.71-2.76\left(\mathrm{t}, 2 \mathrm{H}, \mathrm{C}_{5} \mathrm{CH}_{2}\right.$ or $\left.\mathrm{C}_{6} \mathrm{CH}_{2}\right), 1.99-2.28$ 
(t,2H, $\mathrm{C}_{5} \mathrm{CH}_{2}$ or $\mathrm{C}_{6} \mathrm{CH}_{2}$ ). Anal. Calcd for $\mathrm{C}_{25} \mathrm{H}_{22} \mathrm{~N}_{2} \mathrm{~S}: \mathrm{C}, 78.53 ; \mathrm{H}, 5.76 ; \mathrm{N}, 7.33$. Found: C, 78.46; H, 5.79; N, 7.35\%.

2o $\quad 7.84(\mathrm{~s}, 1 \mathrm{H}, \mathrm{NH}), \quad 6.88-7.43 \quad(\mathrm{~m}, 7 \mathrm{H}, \mathrm{Ar}-\mathrm{H}), \quad 5.62-5.63 \quad\left(\mathrm{~s}, 1 \mathrm{H}, \mathrm{H}_{\mathrm{A}}\right), \quad 2.75-2.84(\mathrm{t}$, $2 \mathrm{H}, \mathrm{C}_{5} \mathrm{CH}_{2}$ or $\left.\mathrm{C}_{6} \mathrm{CH}_{2}\right), 1.96-2.22$ (t, $2 \mathrm{H}, \mathrm{C}_{5} \mathrm{CH}_{2}$ or $\left.\mathrm{C}_{6} \mathrm{CH}_{2}\right), 3.16-3.20(\mathrm{~m}, 1 \mathrm{H}$ of S$\left.\mathrm{CH}_{2}-\mathrm{CH}_{2}-\mathrm{CH}_{2}-\mathrm{CH}_{3}\right), 3.45-3.57$ (m, $1 \mathrm{H}$ of $\left.\mathrm{S}-\mathrm{CH}_{2}-\mathrm{CH}_{2}-\mathrm{CH}_{2}-\mathrm{CH}_{3}\right), 1.29-1.45$ (m,4H, $\underline{\mathrm{CH}}_{2}-\mathrm{CH}_{2}-\mathrm{CH}_{3}$ of S-butyl), 0.85-0.89 (t,3H,- $\mathrm{CH}_{3}$ of S-butyl). Anal. Calcd for $\mathrm{C}_{22} \mathrm{H}_{23} \mathrm{~N}_{2} \mathrm{SCl}$ : $\mathrm{C}$ 63.31; H, 5.51; N, 6.71. Found: $\mathrm{C}, 63.02 ; \mathrm{H}, 5.76 ; \mathrm{N}, 6.10 \%$.

$2 \mathrm{p} \quad 7.88(\mathrm{~s}, 1 \mathrm{H}, \mathrm{NH}), 6.98-7.31(\mathrm{~m}, 8 \mathrm{H}, \mathrm{Ar}-\mathrm{H}), 5.03\left(\mathrm{~s}, 1 \mathrm{H}, \mathrm{H}_{\mathrm{A}}\right), 3.76\left(\mathrm{~s}, 3 \mathrm{H}, \mathrm{OCH}_{3}\right), 2.62-$ 2.75(t, $2 \mathrm{H}, \mathrm{C}_{5} \mathrm{CH}_{2}$ or $\left.\mathrm{C}_{6} \mathrm{CH}_{2}\right), 1.95-2.12\left(\mathrm{t}, 2 \mathrm{H}, \mathrm{C}_{5} \mathrm{CH}_{2}\right.$ or $\left.\mathrm{C}_{6} \mathrm{CH}_{2}\right), 3.20-3.29(\mathrm{~m}, 1 \mathrm{H}$ of S- $\left.\mathrm{CH}_{2}-\mathrm{CH}_{2}-\mathrm{CH}_{2}-\mathrm{CH}_{3}\right), 3.41-3.55\left(\mathrm{~m}, 1 \mathrm{H}\right.$ of $\left.\mathrm{S}-\mathrm{CH}_{2}-\mathrm{CH}_{2}-\mathrm{CH}_{2}-\mathrm{CH}_{3}\right), 1.45-1.60$ (m, $4 \mathrm{H}, \underline{\mathrm{CH}}_{2}-\mathrm{CH}_{2}-\mathrm{CH}_{3}$ of S-butyl), 0.80-0.84 (t,3H,- $\mathrm{CH}_{3}$ of S-butyl). Anal. Calcd for $\mathrm{C}_{23} \mathrm{H}_{26} \mathrm{~N}_{2} \mathrm{SO}$ : C 73.01; H, 6.88; N, 7.41. Found: C, 72.95; H, 6.32; N, 7.10\%.

$2 \mathrm{q} \quad 7.73(\mathrm{~s}, 1 \mathrm{H}, \mathrm{NH}), 7.16-7.34(\mathrm{~m}, 8 \mathrm{H}, \mathrm{Ar}-\mathrm{H}), 5.44\left(\mathrm{~s}, 1 \mathrm{H}, \mathrm{H}_{\mathrm{A}}\right), 2.70-2.79\left(\mathrm{t}, 2 \mathrm{H}, \mathrm{C}_{5} \mathrm{CH}_{2}\right.$ or $\left.\mathrm{C}_{6} \mathrm{CH}_{2}\right), 1.90-2.06$ (t, $2 \mathrm{H}, \mathrm{C}_{5} \mathrm{CH}_{2}$ or $\left.\mathrm{C}_{6} \mathrm{CH}_{2}\right), 2.43\left(\mathrm{~s}, 3 \mathrm{H}, \mathrm{CH}_{3}\right), 3.10-3.22(\mathrm{~m}, 1 \mathrm{H}$ of $\left.\mathrm{S}-\mathrm{CH}_{2}-\mathrm{CH}_{2}-\mathrm{CH}_{2}-\mathrm{CH}_{3}\right), 3.52-3.64$ (m, $1 \mathrm{H}$ of $\left.\mathrm{S}-\mathrm{CH}_{2}-\mathrm{CH}_{2}-\mathrm{CH}_{2}-\mathrm{CH}_{3}\right), 1.31-1.56$

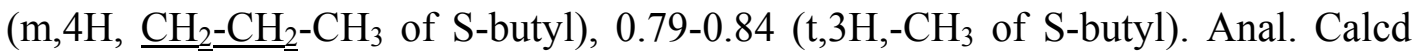
for $\mathrm{C}_{23} \mathrm{H}_{26} \mathrm{~N}_{2} \mathrm{~S}$ : C 76.24; H, 7.18; N, 7.73. Found: $\mathrm{C}, 76.48 ; \mathrm{H}, 7.32 ; \mathrm{N}, 7.55 \%$.

$2 \mathrm{r}$ $11.46(\mathrm{~s}, 1 \mathrm{H}, \mathrm{NH}), \quad 5.40-5.41 \quad\left(\mathrm{~s}, 1 \mathrm{H}, \mathrm{H}_{\mathrm{A}}\right), \quad 5.97-5.98 \quad\left(\mathrm{~s}, 2 \mathrm{H}, \mathrm{O}-\mathrm{CH}_{2}-\mathrm{O}\right), \quad 6.79-7.71$ $(\mathrm{m}, 7 \mathrm{H}, \mathrm{Ar}-\mathrm{H}), 2.09-2.31\left(\mathrm{t}, 2 \mathrm{H}, \mathrm{C}_{5} \mathrm{CH}_{2}\right.$ or $\left.\mathrm{C}_{6} \mathrm{CH}_{2}\right), 2.79-2.83\left(\mathrm{t}, 2 \mathrm{H}, \mathrm{C}_{5} \mathrm{CH}_{2}\right.$ or $\mathrm{C}_{6} \mathrm{CH}_{2}$ ), 3.18-3.25 (m, $1 \mathrm{H}$ of S- $\mathrm{CH}_{2}-\mathrm{CH}_{2}-\mathrm{CH}_{2}-\mathrm{CH}_{3}$ ), 3.56-3.63 (m, $1 \mathrm{H}$ of S- $\mathrm{CH}_{2}-$ $\left.\mathrm{CH}_{2}-\mathrm{CH}_{2}-\mathrm{CH}_{3}\right), 1.34-1.55\left(\mathrm{~m}, 4 \mathrm{H}, \mathrm{CH}_{2}-\mathrm{CH}_{2}-\mathrm{CH}_{3}\right.$ of S-butyl), 0.81-0.85 (t,3H,$\mathrm{CH}_{3}$ of S-butyl) m/z $392(0.39 \%) \mathrm{M}^{+}, 335(100 \%), 178$ (7.18\%), 214 (91.95\%). Anal. Calcd for $\mathrm{C}_{23} \mathrm{H}_{25} \mathrm{~N}_{2} \mathrm{SO}_{2}$ : C, 70.41; H, 6.37; N, 7.14. Found: C, 70.45; H, 6.40; N, $7.18 \%$.

2s $\quad 10.67(\mathrm{~s}, 1 \mathrm{H}, \mathrm{NH}), 7.98(\mathrm{~S}, 1 \mathrm{H}, \mathrm{OH}), 6.61-7.52(\mathrm{~m}, 7 \mathrm{H}, \mathrm{Ar}-\mathrm{H}), 5.03\left(\mathrm{~s}, 1 \mathrm{H}, \mathrm{H}_{\mathrm{A}}\right), 2.47-$ $2.54\left(\mathrm{t}, 2 \mathrm{H}, \mathrm{C}_{5} \mathrm{CH}_{2}\right.$ or $\left.\mathrm{C}_{6} \mathrm{CH}_{2}\right), 1.92-2.21\left(\mathrm{t}, 2 \mathrm{H}, \mathrm{C}_{5} \mathrm{CH}_{2}\right.$ or $\left.\mathrm{C}_{6} \mathrm{CH}_{2}\right), 3.34-3.51(\mathrm{~m}, 1 \mathrm{H}$ of S- $\left.\mathrm{CH}_{2}-\mathrm{CH}_{2}-\mathrm{CH}_{2}-\mathrm{CH}_{3}\right), 3.09-3.18\left(\mathrm{~m}, 1 \mathrm{H}\right.$ of $\left.\mathrm{S}-\mathrm{CH}_{2}-\mathrm{CH}_{2}-\mathrm{CH}_{2}-\mathrm{CH}_{3}\right), 1.23-1.44$ (m, $4 \mathrm{H}, \quad \underline{\mathrm{CH}}_{2}-\mathrm{CH}_{2}-\mathrm{CH}_{3}$ of S-butyl), $0.78-0.83 \quad\left(\mathrm{t}, 3 \mathrm{H},-\mathrm{CH}_{3}\right.$ of S-butyl), $3.70\left(\mathrm{~s}, 3 \mathrm{H}, \mathrm{OCH}_{3}\right)$. Anal. Calcd for $\mathrm{C}_{23} \mathrm{H}_{27} \mathrm{~N}_{2} \mathrm{SO}_{2}: \mathrm{C} 67.48 ; \mathrm{H}, 6.60 ; \mathrm{N}, 6.84$. Found: C, $67.12 ; \mathrm{H}, 6.32 ; \mathrm{N}, 6.96 \%$.

$2 \mathrm{t} \quad 11.96(\mathrm{~s}, 1 \mathrm{H}, \mathrm{NH}), 7.15-7.40(\mathrm{~m}, 9 \mathrm{H}, \mathrm{Ar}-\mathrm{H}), 5.10\left(\mathrm{~s}, 1 \mathrm{H}, \mathrm{H}_{\mathrm{A}}\right), 2.68-2.82\left(\mathrm{t}, 2 \mathrm{H}, \mathrm{C}_{5} \mathrm{CH}_{2}\right.$ or $\mathrm{C}_{6} \mathrm{CH}_{2}$ ), 1.95-2.17 (t, $2 \mathrm{H}, \mathrm{C}_{5} \mathrm{CH}_{2}$ or $\mathrm{C}_{6} \mathrm{CH}_{2}$ ), 3.71-3.73 (m,1H of S- $\mathrm{CH}_{2}-\mathrm{CH}_{2}-$ $\left.\mathrm{CH}_{2}-\mathrm{CH}_{3}\right), 3.26-3.29$ (m, $1 \mathrm{H}$ of S- $\left.\underline{\mathrm{CH}}_{2}-\mathrm{CH}_{2}-\mathrm{CH}_{2}-\mathrm{CH}_{3}\right), 1.23-1.36\left(\mathrm{~m}, 4 \mathrm{H}, \underline{\mathrm{CH}_{2}}=\right.$ $\mathrm{CH}_{2}-\mathrm{CH}_{3}$ of S-butyl), 0.85-0.92 (t,3H,- $\mathrm{CH}_{3}$ of S-butyl). Anal. Calcd for $\mathrm{C}_{22} \mathrm{H}_{24} \mathrm{~N}_{2} \mathrm{~S}$ : C 75.86; H, 6.89; N, 8.04. Found: C, 75.52; H, 6.72; N, 8.33\%. 


\section{Acknowledgements}

We are thankful to Department of Chemistry, Punjabi University, Patiala for providing financial assistance to carry out the research work.

\section{References}

1. Brown, D. J.; Quinazolines, Supplement I; The Chemistry of Heterocyclic Compounds, Vol.55; John Wiley \& Sons: Chichester, 1996.

2. (a) Ravina, A. J. Organic Preparations and Procedures Int. 1973, 5, 174. (b) Stevens, G. Diuretics; Academic Press Inc.: New York, 1963; p12. (c) Hess,H. J.; Cronin,T. H.; Sciabine, A. J. Med. Chem. 1968, 11, 130. (d) Leszkuvszky, C.; Erdely, I.; Tardos, L. Acta. Physiol. Acad. Sci. Hung. 1965, 27, 81. (e) Gupta, C. M.; Hussain, S. T.; Bhaduri, A. P.; Khana, N. M.; Mukherjee, S. K. Nature 1969, 223, 524.

3. (a) Kappe, C. O. "The Biginelli Reaction" In Multicomponent Reactions; Zhu, J.; Bienayme, H.; Eds.; Wiley-VCH: Weinheim, 2005; p 95 (b) Bienayme, H.; Hulme, C.; Oddon, G.; Schmitt, P. Chem. Eur. J., 2000, 6, 3321. (c) Domling, A.; Ugi, I. Angew. Chem. Int. Ed., 2000, 39, 3168.

4. (a) Gupta, R.; Gupta, A.K.; Paul, S.; Kachroo, P.L. Indian J. Chem. 1995, 34B, 61. (b) Varma, S. Green Chem. 1999, 1, 43. (c) Borah, R.; Kalita, D. J.; Sarma, J. C.; Indian J. Chem. 2002, 41B, 1032.

5. Bose, A. K.; Manhas, M. S.; Ghosh, M.; Shah, M.; Raju, V. S.; Bari, S. S.; Newaz, S. N.; Banik,B. K.; Chaudhary, A. G.; Barakat, K. J. J. Org. Chem. 1991, 56, 6968.

6. Pal, R.; Handa, R. N.; Pujari, H. K. Indian J. Chem. 1993, 32B, 929.

7. Pal, R.; Handa, R. N.; Pujari, H. K. Indian J. Chem.1994, 33B, 629.

8. Pathak, P.; Kaur, R.; Kaur, B. ARKIVOC 2006, (xvi),160. 